\title{
A rare coexistence of alopecia areata and lichen planus
}

\section{Funda Tamer ${ }^{1}$, Haldun Umudum²}

\author{
${ }^{1}$ Ufuk University School of Medicine, Department of Dermatology, Ankara, Turkey, ${ }^{2}$ Ufuk University School of Medicine, \\ Department of Pathology, Ankara, Turkey
}

Corresponding author: Dr. Funda Tamer, E-mail: fundatmr@yahoo.com

Sir,

A 45-year-old Caucasian male presented with an 18-month history of pruritic rash in the genital area and hair loss on the forearm for the last 12 months. He stated that he did not receive any treatment for these complaints before. The past medical history included a 5-year history of type 2 diabetes mellitus. The patient was taking $1000 \mathrm{mg}$ of oral metformin therapy twice a day. The family history was unremarkable. Dermatological examination revealed a well demarcated, annular patch of hair loss without atrophy measuring $7 \times 5 \mathrm{~cm}$ in size on the extensor surface of the right forearm. Moreover, multiple, flat, shiny erythematous papules measuring 1 to $5 \mathrm{~mm}$ in size were observed on the glans penis (Fig. 1). The skin biopsy was performed from both lesions to reach a definitive diagnosis. The histopathological evaluation of the alopecic patch revealed perifollicular fibrosis and mild lymphocytic infiltration (Fig. 2). However, histopathological evaluation of the glans penis revealed parakeratosis and mild acanthosis, intense lymphocytic infiltrate in the upper dermis and necrotic basal keratinocytes (Fig. 3). Laboratory tests including complete blood count, chemistry panel, sedimentation rate and C-reactive protein were all in normal limits. Venereal disease research laboratory test, anti-human immunodeficiency virus antibody, anti-hepatitis $\mathrm{C}$ virus antibody and hepatitis B surface antigen were negative. Thus, the patient was diagnosed with alopecia areata and lichen planus based on clinical and histopathological findings. The patient was started on $0.1 \%$ hydrocortisone butyrate ointment twice daily and he was adviced to make a follow-up appointment two weeks later.
Lichen planus is a chronic inflammatory disease of mucosa, hair and nails which usually presents with pruritic, plane, purple and polygonal papules. The etiopathogenesis of lichen planus has not been identified yet. However, it is considered to be a T-cellmediated autoimmune disease in which $\mathrm{CD} 8+\mathrm{T}$

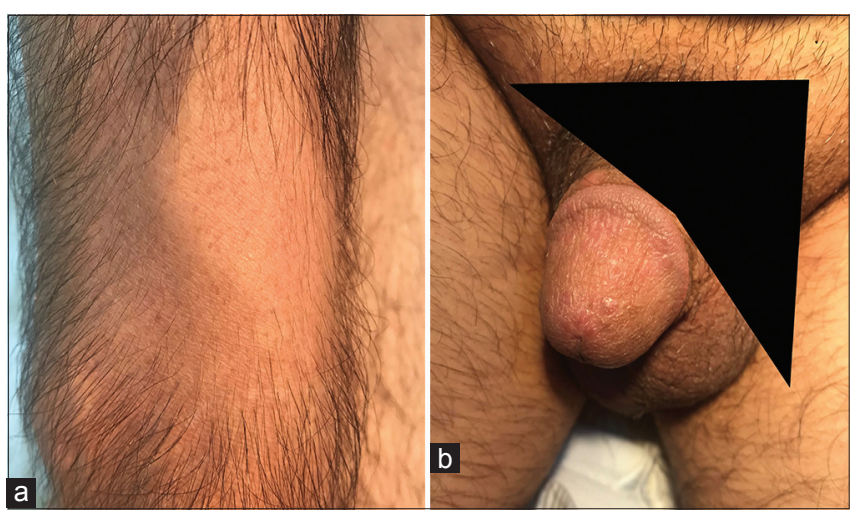

Figure 1: a. Patchy hair loss on the extensor surface of the right forearm b. Multiple erythematous papules on the glans penis.

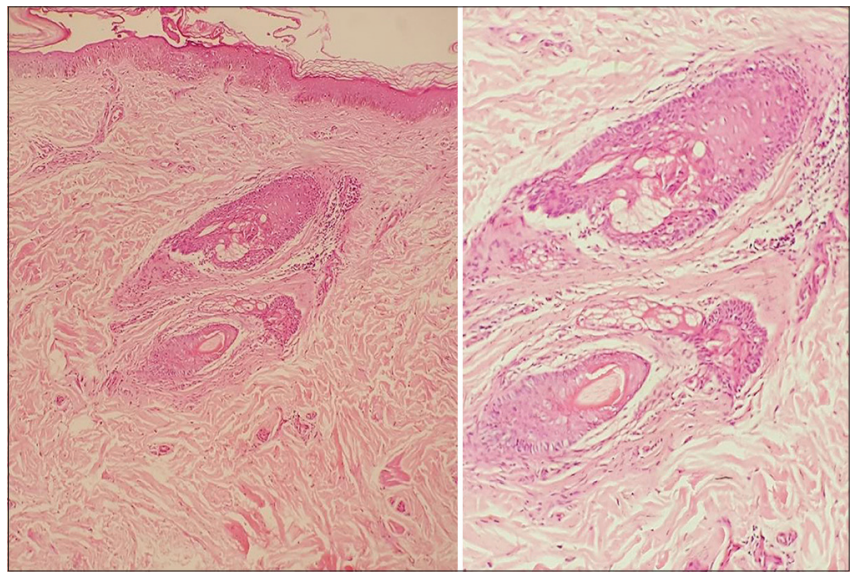

Figure 2: Perifollicular fibrosis and mild lymphocytic infiltration a. H\&Ex4 b. H\&Ex40. 


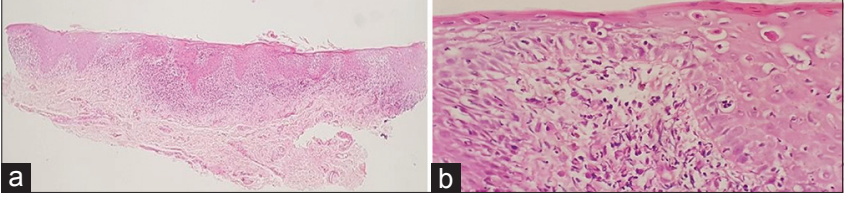

Figure 3: a. Band-like (lichenoid) lymphocytic infiltrate in the upper dermis (H\&Ex2) b. Basal cell vacuolization and necrotic keratinocytes (H\&Ex40)

cells damage basal keratinocytes [1]. Alopecia areata presents with non-scarring, patchy hair loss as a result of destruction of hair bulb by cytotoxic T cells. Alopecia areata is regarded as a skin-restricted autoimmune disease. However, association between alopecia areata, and various inflammatory and autoimmune disorders including lichen planus has been described [2]. Furthermore, Kar et al. and Dhar et al. reported colocalization of lichen planus and alopecia areata, previously $[3,4]$. Lichen planus is characterized by lymphocyte and Langerhans cell infiltartion, and destruction in the basal cell layer of the epidermis whereas, alopecia areata shows perifollicular infiltrates of lymphocytes and Langerhans cells, and follicle destruction. Therefore, it has been suggested that common antigenic determinant may be a triggering factor in the onset of both diseases [4]. Hereby, we present a patient who had alopecia areata and lichen planus at the same time because of its rarity. External surface of the forearm and glans penis were the affected areas. The onset of lichen planus was observed formerly, it was followed by alopecia areata. Our case may help to contribute the literature to determine the correlated etiologic factors in both diseases.

\section{Consent}

The examination of the patient was conducted according to the Declaration of Helsinki principles.

\section{REFERENCES}

1. Chung PI, Hwang CY, Chen YJ, Lin MW, Chen TJ, Hua TC, et al. Autoimmune comorbid diseases associated with lichen planus: a nationwide case-control study. J Eur Acad Dermatol Venereol. 2015;29:1570-5.

2. Garzorz N, Alsisi M, Todorova A, Atenhan A, Thomas J, Lauffer F, et al. Dissecting susceptibility from exogenous triggers: the model of alopecia areata and associated inflammatory skin diseases. J Eur Acad Dermatol Venereol. 2015;29:2429-35.

3. Kar BR, Ebenezer G, Job CK. Colocalisation of alopecia areata and lichen planus. Indian J Dermatol Venereol Leprol. 2004;70:242-3.

4. Dhar S, Dhar S. Colocalization of alopecia areata and lichen planus. Pediatr Dermatol. 1996;13:258-9.

Copyright by Funda Tamer, et al. This is an open-access article distributed under the terms of the Creative Commons Attribution License, which permits unrestricted use, distribution, and reproduction in any medium, provided the original author and source are credited.

Source of Support: Nil, Conflict of Interest: None declared. 